\title{
miR-590-5p targets RMND5A and promotes migration in pancreatic adenocarcinoma cell lines
}

\author{
SIXING CHEN ${ }^{1}$, YU CHEN $^{1}$, YAO WEN $^{1}$, WANWAN CAI ${ }^{1}$, PING ZHU $^{2}$, WUZHOU YUAN $^{1}$, \\ YONGQING LI ${ }^{1}$, XIONGWEI FAN ${ }^{1}$, YONGQI WAN ${ }^{1}$, FANG LI $^{1}$, JIAN ZHUANG ${ }^{2}$, \\ ZHIGANG JIANG $^{1}$, XIUSHAN WU ${ }^{1}$ and YUEQUN WANG ${ }^{1}$
}

\begin{abstract}
${ }^{1}$ The Center for Heart Development, State Key Laboratory of Developmental Biology of Freshwater Fish, College of Life Sciences, Hunan Normal University, Changsha, Hunan 410081; ${ }^{2}$ Guangdong Cardiovascular Institute, Guangdong General Hospital, Guangdong Academy of Medical Sciences, Guangzhou, Guangdong 510100, P.R. China
\end{abstract}

Received September 29, 2020; Accepted March 19, 2021

DOI: $10.3892 / \mathrm{ol} .2021 .12793$

\begin{abstract}
Required for meiotic nuclear division 5 homolog A (RMND5A) functions as an E3 ubiquitin ligase. To date, few studies have investigated the role of RMND5A in cancer. In the present study, the expression levels of RMND5A in multiple types of cancer were analyzed using the Gene Expression Profiling Interactive Analysis platform. The results revealed that RMND5A was highly expressed and associated with overall survival in patients with pancreatic adenocarcinoma (PAAD). A wound-healing assay revealed that RMND5A overexpression significantly increased cell migration in the PAAD cell lines AsPC-1 and PANC-1. In silico analysis predicted that RMND5A was a potential target of microRNA(miR)-590-5p. Further in vitro experiments demonstrated that overexpression of miR-590-5p downregulated the expression levels of RMND5A and decreased the migratory ability of the AsPC-1 and PANC-1 cell lines. In addition, overexpression of miR-590-5p attenuated the promoting effects of RMND5A on the migration of AsPC-1 and PANC-1 cells. The results of the present study may further elucidate the mechanisms underlying PAAD progression and provide novel targets for the treatment of PAAD.
\end{abstract}

\section{Introduction}

Glucose-induced degradation-deficient (GID) genes are required for the glucose-induced degradation of yeast

Correspondence to: Professor Xiushan $\mathrm{Wu}$ or Professor Yuequn Wang, The Center for Heart Development, State Key Laboratory of Developmental Biology of Freshwater Fish, College of Life Sciences, Hunan Normal University, 36 Lushan South Road, Changsha, Hunan 410081, P.R. China

E-mail: xiushanwu@yahoo.com

E-mail: yuequnwang@hunnu.edu.cn

Key words: microRNA-590-5p, cell migration, pancreatic cancer, required for meiotic nuclear division 5 homolog A, prognosis fructose-1,6-bisphosphatase (1). Required for meiotic nuclear division 5 homolog A (RMND5A) is the human ortholog of the yeast gene, Gid2, which contains the Lis homology (LisH), C-terminal to LisH, CT11-RanBPM and E3 typical RING domains (1,2). RMND5A functions as an E3 ubiquitin ligase, which combines with E1 and E2 enzymes to catalyze protein ubiquitination $(1,3)$. Previous studies have reported that RMND5A regulated c-Raf expression and that downregulation of RMND5A inhibited the migration of HeLa cells (4-6). Abnormal expression levels of RMND5A have been found to be associated with poor prognosis in breast cancer (7). However, there have been few reports on the roles of RMND5A in cancer.

MicroRNAs (miRNAs/miRs) are small non-coding RNA molecules, 21-23 nucleotides in length, which can regulate gene expression at the post-transcriptional level by degrading targeted mRNAs or suppressing their translation (8-11). Numerous studies have demonstrated the important roles of miRNAs during angiogenesis and tumorigenesis (12-15). To date, only miR-21 and miR-138 have been reported to target RMND5A $(6,16)$. The aim of the present study was to investigate the differential expression of RMND5A and its prognostic role in multiple types of cancer. The present results revealed a crucial function of RMND5A in pancreatic adenocarcinoma (PAAD) and identified a novel miRNA, miR-590-5p, that targets RMND5A in PAAD cell lines.

\section{Materials and methods}

Cell lines and culture conditions. Two human PAAD cell lines, AsPC-1 and PANC-1, were purchased from the American Type Culture Collection and were cultured in RPMI-1640 medium (Gibco; Thermo Fisher Scientific, Inc.) supplemented with $10 \%$ fetal bovine serum (HyClone; Cytiva) at $37^{\circ} \mathrm{C}$ in a humidified incubator with $5 \% \mathrm{CO}_{2}$.

Identification of potential miRNAs targeting RMND5A. Four miRNA-target gene databases were used to predict the miRNAs targeting RMND5A: TargetScan (http://www.targetscan.org/), miRDB (http://www.mirdb. org/), miRcode (http://www.mircode.org/) and TarBase v8 (http://www.microrna.gr/tarbase). In addition, we further 
evaluated their possible interactions by using starBase (http://starbase.sysu.edu.cn/).

Cell transfection. miR-590-5p mimics (5'GAGCUUAUUCAU AAAAGU-GCAG-3') and negative control (5'CGGUACGAU CGCGGCGGGAUAUC-3') were synthesized by Guangzhou RiboBio Co., Ltd., and transfected into the PAAD cell lines using Lipofectamine $^{\circledR} 2000$ (Invitrogen; Thermo Fisher Scientific, Inc.) at the concentration of $100 \mathrm{nM}$. After transfection for $48 \mathrm{~h}$, reverse transcription-quantitative PCR (RT-qPCR) analysis was performed to detect the expression levels of miR-590-5p. The plasmid for overexpressing human RMND5A was constructed using the pHS-AVC (pLV-hef1a-mScarlet-P2A-neo-WPRE -CMV) vector (Beijing Syngentech Co., Ltd.), according to the human RMND5A gene sequence in Genbank (NM_022780.4). HEK293 (CRL-1573, ATCC) cultured in 6-cm dishes were used to pack viral particles for RMND5A overexpression. Briefly, $27 \mu \mathrm{l} \mathrm{FuGENE}{ }^{\circledR} 6$ (Promega Corporation), $4 \mu \mathrm{g}$ of recombinant plasmids or empty vector as control, $3 \mu \mathrm{g}$ PAX2 (cat. no. 12260; Addgene, Inc.) and $2 \mu \mathrm{g} \mathrm{VSVG} \mathrm{(cat.} \mathrm{no.} \mathrm{8454;} \mathrm{Addgene} \mathrm{Inc.)}$ were mixed in 300 ul OPTI-MEM (Gibco; Thermo Fisher Scientific, Inc.), followed by being dropped into $6-\mathrm{cm}$ dish. Then, the cells were placed back in the incubator at $37^{\circ} \mathrm{C}$. After $6 \mathrm{~h}$, all the medium was replaced by pre-warmed DMEM medium (Gibco; Thermo Fisher Scientific, Inc.) supplemented with $10 \%$ fetal bovine serum. After $48 \mathrm{~h}, \sim 4 \mathrm{ml}$ of the lentiviral particles in culture media were collected. For infection, $1 \mathrm{ml}$ of the lentiviral particles and $10 \mu \mathrm{g} / \mathrm{ml}$ polybrene (Sigma-Aldrich; Merck $\mathrm{KGaA}$ ) were used to infect 0.5 million cells at $37^{\circ} \mathrm{C}$ for $4 \mathrm{~h}$. After $4 \mathrm{~h}$, more prewarmed medium was added for culturing at $37^{\circ} \mathrm{C}$. After $48 \mathrm{~h}, \mathrm{RT}-\mathrm{qPCR}$ analysis was performed to detect the mRNA expression levels of RMND5A.

$R T-q P C R$. Total RNA was extracted either using the mirVana miRNA kit (Ambion; Thermo Fisher Scientific, Inc.) or the E.Z.N.A ${ }^{\circledR}$ Total RNA kit (Omega Bio-Tek, Inc.). For mRNA detection, 1,000 ng RNA was reverse transcribed into cDNA using qScript cDNA Synthesis kit (cat. no. 95047; Quantabio) according to the manufacturer's protocol and quantified using the Fast Start Universal SYBRGreen master mix (cat. no. 4913850001; Merck KGaA). For miRNA detection, $250 \mathrm{ng}$ RNA was reverse-transcribed into cDNA and quantified using the Mir- $\mathrm{X}^{\mathrm{TM}}$ miRNA qRT-PCR TB Green ${ }^{\mathrm{TM}}$ kit (cat. no. 638314; Takara Biotechnology Co., Ltd.). GAPDH and U6 were used as the internal control for mRNA and miRNA, respectively. The QuantStudio 6Flex Real-Time PCR System (Applied Biosystems; Thermo Fisher Scientific, Inc.) was used for qPCR. The thermocycling conditions used were as follows: Pre-denaturation at $95^{\circ} \mathrm{C}$ for $1 \mathrm{~min}$; 40 cycles of denaturation at $95^{\circ} \mathrm{C}$ for $15 \mathrm{sec}$; annealing at $60^{\circ} \mathrm{C}$ for $15 \mathrm{sec}$; and extension at $72^{\circ} \mathrm{C}$ for $30 \mathrm{sec}$ followed by melting curve analysis. For quantification, $2^{-\Delta \Delta \mathrm{Cq}}$ method (17) was used and the primer sequences are listed in Table I.

Western blot analysis. Total protein was extracted from the AsPC-1 cells using the radioimmunoprecipitation assay (RIPA) lysis buffer (Beyotime Institute of Biotechnology). The Pierce BCA Protein Assay kit (Thermo Fisher Scientific, Inc.) was used to quantify the total extracted protein according to the manufacturer's protocol. A total of $30 \mu \mathrm{g}$ of proteins were separated using 10\% SDS-PAGE and transferred onto PVDF membranes (EMD Millipore) at room temperature (RT). The membranes were subsequently incubated with rabbit polyclonal anti-RMND5A (1:1,000; cat. no. ab254902; Abcam) and rabbit polyclonal anti-GAPDH antibodies (1:1,000; cat. no. 10494-1-AP; ProteinTech Group, Inc.) overnight at $4^{\circ} \mathrm{C}$. Then, the membranes were incubated with goat polyclonal anti-rabbit IgG secondary antibody (1:1,000; cat. no. ab6721; Abcam) for $1 \mathrm{~h}$ at RT. The protein expression levels were measured using an enhanced chemiluminescence kit (Pierce; Thermo Fisher Scientific, Inc.).

Wound healing assay. The human PAAD cell lines AsPC-1 and PANC-1 were seeded in a 6-well plate at $1 \times 10^{6}$ cells/well. When the cells reached $80-90 \%$ confluence, the cell monolayer was scraped with a $200-\mu 1$ pipette tip. After washing with PBS, the cells were cultured for another $24 \mathrm{~h}$. The medium used for this assay was serum-free DMEM. The migrated cells were observed using a phase-contrast light microscope at x10 objective magnification (Olympus Corporation).

Statistical analysis. Data are presented as mean \pm SD and analysed using GraphPad Prism 8 (GraphPad Software Inc.). A total of 3 biological replicates and 3 technical repeats were performed. For the comparison of two groups, Student's t-test was used. One-way ANOVA followed by Tukey's multiple comparisons test was performed when comparing more than two groups. $\mathrm{P}<0.05$ was considered to indicate a statistically significant difference.

\section{Results}

RMND5A is highly expressed and associated with overall survival in patients with PAAD. To investigate the role of RMND5A in cancer, the expression levels of RMND5A in 31 types of cancer were analyzed using the Gene Expression Profiling Interactive Analysis platform (http://gepia. cancer-pku.cn). As shown in Fig. $1 \mathrm{~A}$ and B, the expression levels of RMND5A were significantly higher in PAAD, stomach adenocarcinoma (STAD) and thymoma (THYM) tissues compared with that in normal tissues. Notably, a lower RMND5A expression was associated with better overall survival in patients with PAAD (Fig. 1C). However, in patients with STAD and THYM, RMND5A expression levels were not significantly associated with overall survival (Fig. 1C).

RMND5A promotes migration in PAAD cell lines. Based on the aforementioned data, the effects of RMND5A overexpression were investigated in PAAD cell lines to determine its function. The transfection efficiency of the RMND5A overexpression plasmid was confirmed using RT-qPCR and western blot analyses (Fig. 2A and B). Notably, it was found that overexpression of RMND5A significantly increased cell migration in both the AsPC-1 and PANC-1 cell lines (Fig. 2C and D).

miR-590-5p targets the RMND5A gene. To predict the potential miRNAs that target RMND5A, in silico analysis was performed by using the TargetScan, miRDB, miRcode and TarBase databases. The overlap analysis identified six potential miRNAs, namely miR-590-5p, miR-30a-5p, miR-27a-3p, miR-148b-3p, miR-30b-5p, and miR-30e-5p (Fig. 3A). In addition, the results of the targeting sites, Cross-linking 
Table I. Primer sequences used for reverse transcription-quantitative PCR analysis.

\begin{tabular}{lll}
\hline Gene & \multicolumn{1}{c}{ Forward primer $\left(5^{\prime}-3^{\prime}\right)$} & \multicolumn{1}{c}{ Reverse primer $\left(5^{\prime}-3^{\prime}\right)$} \\
\hline miR-590-5p & GAGCTTATTCATAAAAGT & TCCACGACACGCACTGGATACGAC \\
U6 & CTCGCTTCGGCAGCACA & AACGCTTCACGAATTTGCGT \\
RMND5A & AATGAGGTGATGGTGGAGCA & GCATTTCCCGGTTTGACACT \\
GAPDH & GGAGCGAGATCCCTCCAAAAT & GGCTGTTGTCATACTTCTCATGG
\end{tabular}

miR, microRNA; RMND5A, required for meiotic nuclear division 5 homolog A.
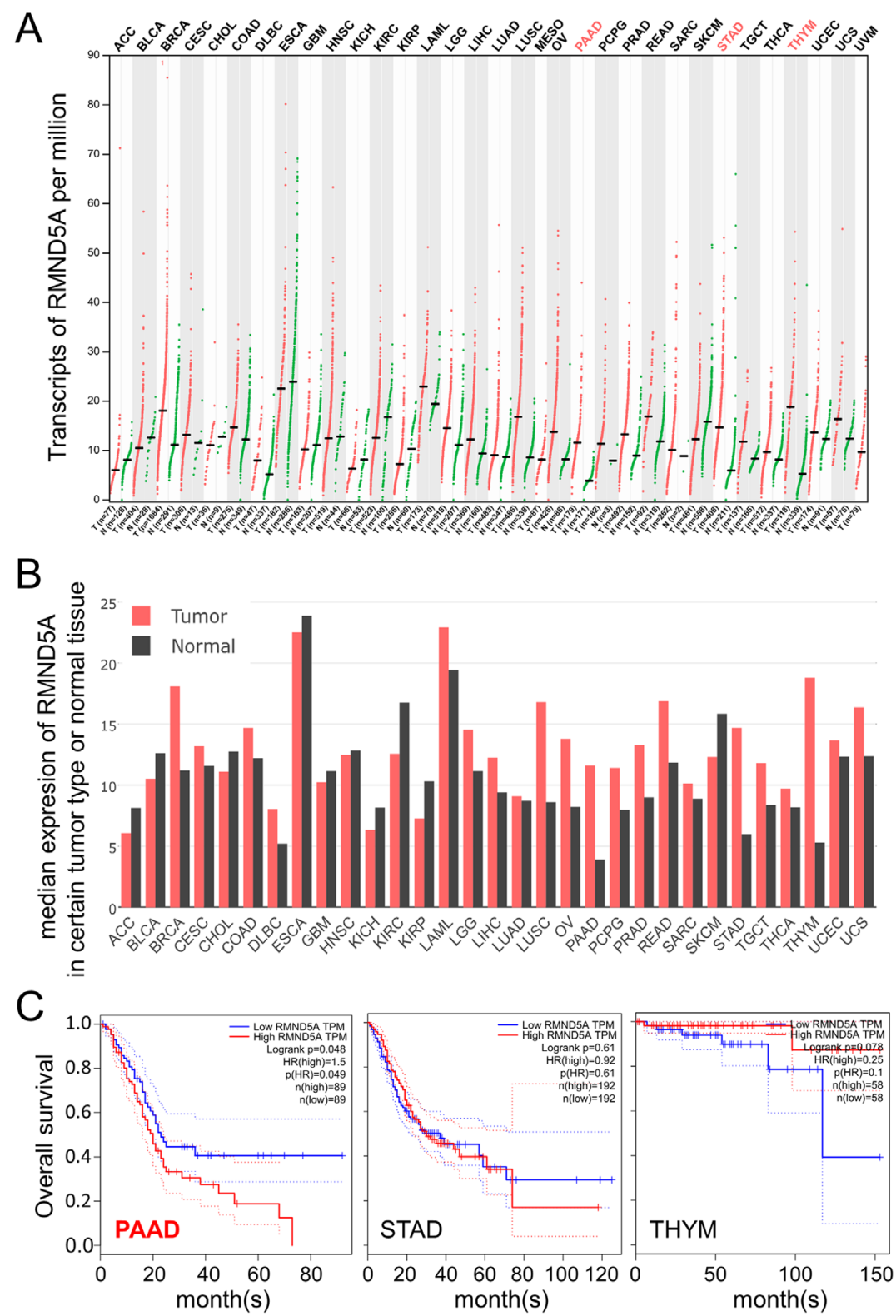

Figure 1. RMND5A is highly expressed in the tumor tissues of patients with PAAD and is associated with overall survival. Data in the Gene Expression Profiling Interactive Analysis platform (http://gepia.cancer-pku.cn) were analyzed in respect to RMND5A expression. (A) Transcripts per million and (B) median expression of RMND5A are plotted in multiple types of cancer. (C) Association between RMND5A expression levels and overall survival in patients with PAAD, STAD and THYM. RMND5A, required for meiotic nuclear division 5 homolog A; ACC, adenoid cystic carcinoma; BLCA, bladder urothelial carcinoma; BRCA, breast cancer; CESC, cervical squamous cell carcinoma and endocervical adenocarcinoma; CHOL, cholangiocarcinoma; COAD, colon adenocarcinoma; DLBC, diffuse large B-cell lymphoma; ESCA, esophageal carcinoma; GBM, glioblastoma; HNSC, head and neck squamous cell carcinoma; KICH, kidney chromophobe; KIRC, kidney renal clear cell carcinoma; KIRP, kidney renal papillary cell carcinoma; LAML, acute myeloid leukemia; LGG, brain lower grade glioma; LIHC, hepatocellular carcinoma; LUAD, lung adenocarcinoma; LUSC, lung squamous cell carcinoma; MESO, mesothelioma; OV, ovarian serous cystadenocarcinoma; PAAD, pancreatic adenocarcinoma; PCPG, pheochromocytoma and paraganglioma; PRAD, prostate adenocarcinoma; READ, rectum adenocarcinoma; SARC, sarcoma; SKCM, skin cutaneous melanoma; STAD, stomach adenocarcinoma; TGCT, testicular germ cell tumors; THCA, thyroid carcinoma; THYM, thymoma; UCEC, uterine corpus endometrial carcinoma; UCS, uterine carcinosarcoma; UVM, uveal melanoma. 
A

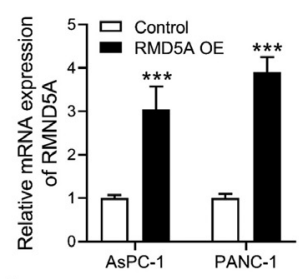

C

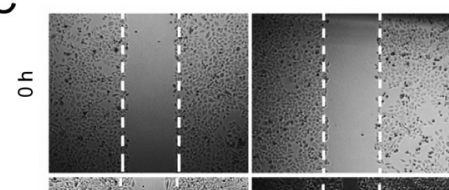

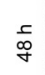

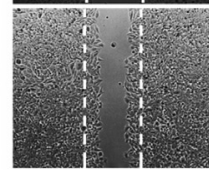

AsPC-1 Control AsPC-1 RMND5A OE

$\mathrm{D}$

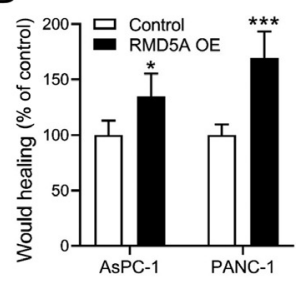

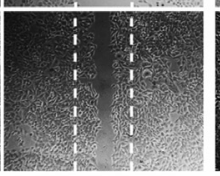

B
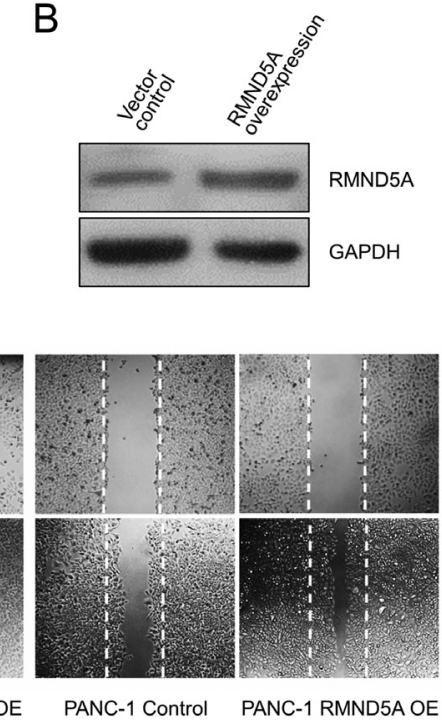

PANC-1 Control PANC-1 RMND5A OE

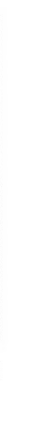

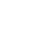



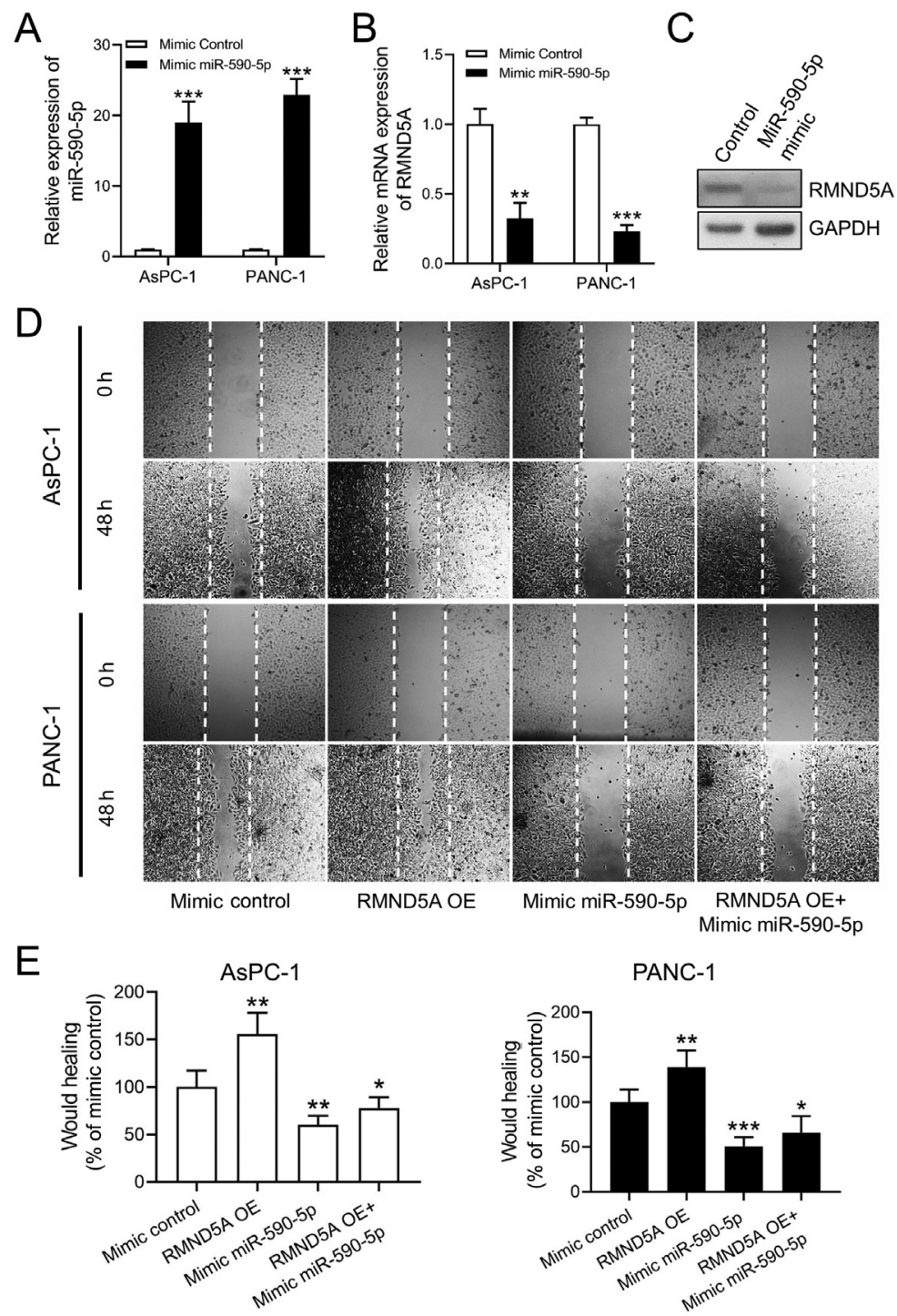

Figure 4. Overexpression of miR-590-5p inhibits RMND5A expression and pancreatic adenocarcinoma cell migration. (A) Expression levels of miR-590-5p and (B) RMND5A mRNA following transfection with miR-590-5p mimics in AsPC-1 and PANC-1 cells. (C) Western blot analysis was used to determine the protein levels of RMND5A in the AsPC-1 cells following transfection with miR-590-5p mimics. (D) Wound healing assay was used to determine the migration of AsPC-1 and PANC-1 cells following transfection with miR-590-5p mimics, with or without overexpression of RMND5A. Representative images at 0 and $48 \mathrm{~h}$ are shown (magnification, $\mathrm{x} 10$ ). (E) Quantification of the results of the wound healing assay. Data are presented as the mean \pm SD. "P<0.05, ${ }^{* *} \mathrm{P}<0.01$ and ${ }^{* * *} \mathrm{P}<0.001$. miR, microRNA; RMND5A, required for meiotic nuclear division 5 homolog A; OE, overexpression.

immunoprecipitation (ClipSeq) PeakCluster and ReadNumber of miRNA-RMND5A interaction in StarBase were checked, which revealed that miR-590-5p was among the top hits. The 3' untranslated region (UTR) of both miR-590-5p and RMND5A were highly conserved among mammals (Fig. 3B). Finally, the mature miR-590-5p seeding region exhibited a perfect alignment with the RMND5A 3'UTR (Fig. 3C).

Overexpression of miR-590-5p inhibits RMND5A expression and cell migration in PAAD cell lines. To confirm the hypothesis that miR-590-5p targets the RMND5A gene, miR-590-5p mimics were transfected into the PAAD cell lines. The transfection efficiency of miR-590-5p mimics was confirmed using RT-qPCR (Fig. 4A). Following transfection with miR-590-5p mimics, the AsPC-1 and PANC-1 cell lines exhibited significantly decreased RMND5A expression levels (Fig. 4B and C). The wound healing assay demonstrated that miR-590-5p mimics decreased the migration ability of the AsPC-1 and PANC-1 cells (Fig. 4D and 4E). Furthermore, miR-590-5p mimics attenuated the promoting effects of RMND5A overexpression on the migration of the PAAD cells (Fig. 4D and E).

\section{Discussion}

PAAD is a common gastrointestinal malignancy with poor prognosis $(18,19)$. Only few effective drugs have been developed for the treatment of PAAD (19). The 5-year survival rate is $<5 \%$ and the majority of patients with PAAD succumb to metastatic disease $(18,20)$. It is well-known that distant metastasis is associated with the migration of tumor cells $(19,21)$. Therefore, it is important to elucidate the molecular pathogenic processes underlying the migration of PAAD cells. Previous studies have demonstrated that RMND5A is important for cell migration and adhesion $(5,7)$. RMND5A was also found to be involved 
in the metastasis of primary tumors $(5,22)$. However, to the best of our knowledge, the association between RMND5A and PAAD has not been investigated. In the present study, it was observed that the RMND5A expression levels were significantly upregulated in PAAD tissues compared with the control normal tissues. High levels of RMND5A in patients with PAAD were associated with poor overall survival. Mechanistically, RMND5A was found to have a key role in the migration of PAAD cells, with RMND5A overexpression significantly increasing the PAAD cell migratory ability. The results of the present study indicated that RMND5A may be a novel prognostic biomarker for patients with PAAD.

In silico analysis identified miR-590-5p as a potential miRNA that may target RMND5A. Notably, the effects of miR-590-5p on tumorigenesis are controversial. Numerous studies have demonstrated that miR-590-5p functions as a tumor suppressor in breast cancer, non-small cell lung cancer and tongue squamous cell carcinoma (23-25). miR-590-5p may also inhibit the migration or proliferation of breast cancer and hepatocellular carcinoma cells by targeting the Wnt signaling pathway $(26,27)$. In addition, miR-590-5p may inhibit the proliferation of malignant melanoma cells and suppress the chemoresistance of hepatocellular carcinoma cells by targeting Yes1 associated transcriptional regulator $(28,29)$. By contrast, it has also been reported that miR-590-5p may promote cell proliferation and invasion in cervical cancer, renal cell carcinoma and endometrial cancer (30-33). In addition, miR-590-5p may promote malignant behaviors in vulvar squamous cell carcinoma and hepatocellular carcinoma cells by targeting TGF $\beta$ receptor $2(34,35)$. To the best of our knowledge, the present study is the first to report that miR-590-5p inhibited the migration of PAAD cells by decreasing RMND5A expression. Furthermore, overexpression of miR-590-5p attenuated the promoting effects of RMND5A on the migration of PAAD cells. However, as with majority of studies, the design of the current study is subject to limitations. There are two major limitations in this study that could be addressed in future research. Firstly, the specific interaction between RMND5A and miR-590-5p should be further confirmed in the future with more experiments, such as dual-luciferase reporter assay. Secondly, the role of RMND5A in other biological functions associated with PAAD, such as proliferation, invasion and metastasis, requires further investigation.

In summary, to the best of our knowledge, the present study was the first to report the increased expression levels of RMND5A in PAAD tissues and its association with poor overall survival. Functionally, RMND5A was demonstrated to promote the migration of PAAD cells in vitro. In addition, miR-590-5p may inhibit the migration of PAAD cells by targeting RMND5A. These results may shed new light on the mechanisms underlying PAAD progression and provide novel targets for the treatment of PAAD.

\section{Acknowledgements}

Not applicable.

\section{Funding}

The present study was funded by the National Natural Science Foundation of China (grant nos. 31872315 and 81670290), the Cooperative Innovation Center of Engineering and New Products for Developmental Biology of Hunan Province (grant no. 2013-448-6), the National Key Research and Development Program of China (grant no. 2018YFA0108700), the NSFC Projects of International Cooperation and Exchanges (grant. no. 81720102004), the Research Team Project of Natural Science Foundation of Guangdong Province of China (grant no. 2017A030312007), the Science and Technology Planning Projects of Guangdong Province of China (grant no. 2019B020230003), the Special Project of Dengfeng Program of Guangdong Provincial People's Hospital (grant no. DFJH201802) and the Key Program of Guangzhou Science Research Plan (grant no. 805212639211).

\section{Availability of data and materials}

The datasets used and/or analyzed during the present study are available from the corresponding author on reasonable request.

\section{Author's contributions}

SC contributed to the design of the study, data acquisition and analysis, and drafted the manuscript. YC and YoW performed data acquisition and analysis. WC, PZ, WY, YL, XF, YaW, FL and JZ performed data analysis. ZJ, XW and YuW contributed to the design of the experiments and critically revised the manuscript. SC and YC have seen and can confirm the authenticity of the raw data. All authors have read and approved the final manuscript and agree to be accountable for all aspects of the study.

\section{Ethics approval and consent to participate}

Not applicable.

\section{Patient consent for publication}

Not applicable.

\section{Competing interests}

All the authors declare that they have no competing interests.

\section{References}

1. Pfirrmann T, Villavicencio-Lorini P, Subudhi AK, Menssen R, Wolf DH and Hollemann T: RMND5 from Xenopus laevis is an E3 ubiquitin-ligase and functions in early embryonic forebrain development. PLoS One 10: e0120342, 2015.

2. Maitland MER, Onea G, Chiasson CA, Wang X, Ma J, Moor SE, Barber KR, Lajoie GA, Shaw GS and Schild-Poulter C: The mammalian CTLH complex is an E3 ubiquitin ligase that targets its subunit muskelin for degradation. Sci Rep 9: 9864, 2019.

3. van Wijk SJ, de Vries SJ, Kemmeren P, Huang A, Boelens R, Bonvin AM and Timmers HT: A comprehensive framework of E2-RING E3 interactions of the human ubiquitin-proteasome system. Mol Syst Biol 5: 295, 2009.

4. McTavish CJ, Berube-Janzen W, Wang X, Maitland MER, Salemi LM, Hess DA and Schild-Poulter C: Regulation of c-Raf Stability through the CTLH Complex. Int J Mol Sci 20: 934, 2019.

5. Huffman N, Palmieri D and Coppola V: The CTLH complex in cancer cell plasticity. J Oncol 2019: 4216750, 2019. 
6. Li J, Chen Y, Qin X, Wen J, Ding H, Xia W, Li S, Su X, Wang W, $\mathrm{Li} \mathrm{H}$, et al: miR-138 downregulates miRNA processing in HeLa cells by targeting RMND5A and decreasing Exportin-5 stability. Nucleic Acids Res 42: 458-474, 2014.

7. Liu H and Ye H: Screening of the prognostic targets for breast cancer based co-expression modules analysis. Mol Med Rep 16: 4038-4044, 2017.

8. Bartel DP: MicroRNAs: Genomics, biogenesis, mechanism, and function. Cell 116: 281-297, 2004.

9. Djuranovic S, Nahvi A and Green R: A parsimonious model for gene regulation by miRNAs. Science 331: 550-553, 2011.

10. Filipowicz W, Bhattacharyya SN and Sonenberg N: Mechanisms of post-transcriptional regulation by microRNAs: Are the answers in sight? Nat Rev Genet 9: 102-114, 2008.

11. Kusenda B, Mraz M, Mayer J and Pospisilova S: MicroRNA biogenesis, functionality and cancer relevance. Biomed Pap Med Fac Univ Palacky Olomouc Czech Repub 150: 205-215, 2006.

12. Brennecke J, Hipfner DR, Stark A, Russell RB and Cohen SM: Bantam encodes a developmentally regulated microRNA that controls cell proliferation and regulates the proapoptotic gene hid in Drosophila. Cell 113: 25-36, 2003.

13. Chen CZ, Li L, Lodish HF and Bartel DP: MicroRNAs modulate hematopoietic lineage differentiation. Science 303: 83-86, 2004.

14. Iorio MV and Croce CM: MicroRNA dysregulation in cancer: Diagnostics, monitoring and therapeutics. A comprehensive review. EMBO Mol Med 4: 143-159, 2012.

15. Lim LP, Lau NC, Garrett-Engele P, Grimson A, Schelter JM, Castle J, Bartel DP, Linsley PS and Johnson JM: Microarray analysis shows that some microRNAs downregulate large numbers of target mRNAs. Nature 433: 769-773, 2005.

16. Chang JT, Wang F, Chapin W and Huang RS: Identification of MicroRNAs as breast cancer prognosis markers through the cancer genome atlas. PLoS One 11: e0168284, 2016.

17. Livak KJ and Schmittgen TD: Analysis of relative gene expression data using real-time quantitative PCR and the 2(-Delta Delta C(T)) Method. Methods 25: 402-408, 2001.

18. Zhu K, Zhang Z, Zhang H, Wang Z and Wang F: miR-142-3p targeting NUCKS1 inhibits proliferation and invasion of pancreatic cancer cells. Artif Cells Nanomed Biotechnol 48: 415-424, 2020.

19. Yamauchi A, Yamamura M, Katase N, Itadani M, Okada N, Kobiki K, Nakamura M, Yamaguchi Y and Kuribayashi F: Evaluation of pancreatic cancer cell migration with multiple parameters in vitro by using an optical real-time cell mobility assay device. BMC Cancer 17: 234, 2017.

20. Siegel RL, Miller KD and Jemal A: Cancer statistics, 2017. CA Cancer J Clin 67: 7-30, 2017.

21. Roussos ET, Condeelis JS and Patsialou A: Chemotaxis in cancer. Nat Rev Cancer 11: 573-587, 2011.

22. Lambert AW, Pattabiraman DR and Weinberg RA: Emerging biological principles of metastasis. Cell 168: 670-691, 2017.

23. Xu BB, Gu ZF, Ma M, Wang JY and Wang HN: MicroRNA-590-5p suppresses the proliferation and invasion of non-small cell lung cancer by regulating GAB1. Eur Rev Med Pharmacol Sci 22: $5954-5963,2018$
24. Xu Y, Han T, Zhu Y and Chen Q: miR-590-5P inhibits the progression of tongue squamous cell carcinoma by targeting FasL. Int J Clin Exp Pathol 10: 11880-11887, 2017.

25. Yan M, Ye L, Feng X, Shi R, Sun Z, Li Z and Liu T: MicroRNA-590-3p inhibits invasion and metastasis in triple-negative breast cancer by targeting Slug. Am J Cancer Res 10: 965-974, 2020.

26. Gao J, Yu SR, Yuan Y, Zhang LL, Lu JW, Feng JF and Hu SN: MicroRNA-590-5p functions as a tumor suppressor in breast cancer conferring inhibitory effects on cell migration, invasion, and epithelial-mesenchymal transition by downregulating the Wnt- $\beta$-catenin signaling pathway. J Cell Physiol 234: 1827-1841, 2019.

27. Shan X, Miao Y, Fan R, Qian H, Chen P, Liu H, Yan X, Li J and Zhou F: miR-590-5P inhibits growth of HepG2 cells via decrease of S100A10 expression and Inhibition of the Wnt pathway. Int J Mol Sci 14: 8556-8569, 2013.

28. Chen M, Wu L, Tu J, Zhao Z, Fan X, Mao J, Weng Q, Wu X, Huang L, Xu M and Ji J: miR-590-5p suppresses hepatocellular carcinoma chemoresistance by targeting YAP1 expression. EBioMedicine 35: 142-154, 2018.

29. Mou K, Liu B, Ding M, Mu X, Han D, Zhou Y and Wang LJ: lncRNA-ATB functions as a competing endogenous RNA to promote YAP1 by sponging miR-590-5p in malignant melanoma. Int J Oncol 53: 1094-1104, 2018.

30. Chu Y, Ouyang Y, Wang F, Zheng A, Bai L, Han L, Chen Y and Wang H: MicroRNA-590 promotes cervical cancer cell growth and invasion by targeting CHL1. J Cell Biochem 115: 847-853, 2014.

31. Xiao X, Tang C, Xiao S, Fu C and Yu P: Enhancement of proliferation and invasion by MicroRNA-590-5p via targeting PBRM1 in clear cell renal carcinoma cells. Oncol Res 20: 537-544, 2013.

32. Wang L, Wei WQ, Wu ZY and Wang GC: MicroRNA-590-5p regulates cell viability, apoptosis, migration and invasion of renal cell carcinoma cell lines through targeting ARHGAP24. Mol Biosyst 13: 2564-2573, 2017.

33. Zhu M, Zhou X, Zhang J and Zhang Y: MicroRNA-590-5p promotes cell survival in endometrioid endometrial cancer by suppressing tumor suppressor PTEN. Int J Clin Exp Pathol 10: 7836-7846, 2017

34. Jiang X, Xiang G, Wang Y, Zhang L, Yang X, Cao L, Peng H, Xue $P$ and Chen D: MicroRNA-590-5p regulates proliferation and invasion in human hepatocellular carcinoma cells by targeting TGF- $\beta$ RII. Mol Cells 33: 545-551, 2012.

35. Yang $\mathrm{X}$ and $\mathrm{Wu} \mathrm{X}$ : miRNA expression profile of vulvar squamous cell carcinoma and identification of the oncogenic role of miR-590-5p. Oncol Rep 35: 398-408, 2016.

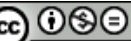

This work is licensed under a Creative Commons Attribution-NonCommercial-NoDerivatives 4.0 International (CC BY-NC-ND 4.0) License. 